\title{
On the Missing Energy Puzzle of Tidal Disruption Events
}

\author{
Wenbin $\mathrm{Lu}^{1,2}$ (1) and Pawan Kumar ${ }^{2}$ \\ ${ }^{1}$ TAPIR, Walter Burke Institute for Theoretical Physics, Mail Code 350-17, Caltech, Pasadena, CA 91125, USA; wenbinlu@caltech.edu \\ ${ }^{2}$ Department of Astronomy, University of Texas at Austin, Austin, TX 78712, USA \\ Received 2018 June 3; revised 2018 July 16; accepted 2018 July 19; published 2018 September 28
}

\begin{abstract}
For the majority of tidal disruption event (TDE) candidates, the observed energy in the optical/near-UV bands is of order $10^{51} \mathrm{erg}$. We show that this observed energy is smaller than the minimum bolometric energy for the radiative inefficient accretion flow model by a factor of 10-100. We argue that this discrepancy is because the majority of the energy released is in the extreme-UV (EUV) band and/or in the form of relativistic jets beamed away from the Earth. The EUV scenario is supported by existing mid-infrared data and should be further tested by future dust reverberation observations. The jet scenario is disfavored by radio observations of ASASSN-14li but may still be viable for other TDE candidates. We also provide evidence that, at least for some TDEs, most of the missing energy (in the EUV and/or in the form of jets) is released within a few times the orbital period of the most tightly bound material $P_{\min }$, which means (1) the circularization of the fallback stream may occur rapidly and (2) the luminosity of the accretion flow or the jet power may not be capped near the Eddington level when the fallback rate is super-Eddington. For most other TDEs, this energy-release timescale is currently not strongly constrained.
\end{abstract}

Key words: galaxies: nuclei - infrared: ISM - methods: analytical

\section{Introduction}

A tidal disruption event (TDE) occurs when a star is shredded by the tidal gravity of a supermassive black hole (BH) with mass $M \lesssim 10^{8} M_{\odot}$ (Rees 1988). To conserve the total angular momentum, roughly half of the star is ejected to infinity, and the other half is left in bound elliptical orbits. The bound materials, after passing the apocenters of their orbits, fall back toward the $\mathrm{BH}$ and generate bright multiwavelength emission. Many TDE candidates have been discovered by transient surveys in the X-ray (e.g., Komossa \& Bade 1999; Donley et al. 2002; Esquej et al. 2008; Saxton et al. 2012), UV (e.g., Gezari et al. 2008, 2009), and optical bands (e.g., van Velzen et al. 2011; Gezari et al. 2012; Arcavi et al. 2014; Vinkó et al. 2015; Holoien et al. 2016a). As opposed to TDE candidates that typically show a thermal spectrum, a few events have been characterized by highly variable, nonthermal (power-law) $\gamma$-/X-ray emission, which is likely generated by relativistic jets pointing toward the Earth (Bloom et al. 2011; Burrows et al. 2011; Zauderer et al. 2011; Cenko et al. 2012; Brown et al. 2015).

Thermal TDE candidates are usually selected from large samples of transient sources by eliminating the by far more common flares from variable AGNs (according to the host spectrum) and supernovae (according to the light-curve shape, temperature evolution, and the distance to the galactic center). However, these methods of selection do not guarantee the purity of the final TDE sample. For instance, supernovae from nuclear star clusters may occur at a rate of $\sim 10^{-4}$ galaxy $^{-1} \mathrm{yr}^{-1}$ for Milky-Way-like galaxies (Zubovas et al. 2013), whereas the measured rate of the TDE candidates is $10^{-4}-10^{-5}$ galaxy $^{-1} \mathrm{yr}^{-1}$ (Donley et al. 2002; van Velzen \& Farrar 2014; Holoien et al. 2016a; Hung et al. 2017b; van Velzen 2018). In this paper, we make the assumption that at least some of these candidates are actual TDEs; this is motivated by the theoretical expectation of the TDE rate being $10^{-4}-10^{-5}$ galaxy $^{-1} \mathrm{yr}^{-1}$ (Magorrian \& Tremaine 1999; Wang
\& Merritt 2004; Stone \& Metzger 2016). This is also supported by the cutoff in the TDE rate for BH masses above $\sim 10^{8} M_{\odot}$ due to the direct capture of stars by the event horizon (van Velzen 2018).

In the generic picture of a TDE, general relativistic apsidal precession causes the fallback stream to self-intersect, and then the shocks at the collision point convert the kinetic energy of the upstream gas into thermal energy. The angular momentum of the stream is redistributed downstream of the shock such that the orbital eccentricity is reduced and viscous accretion may proceed efficiently (e.g., Evans \& Kochanek 1989; Kochanek 1994; Rosswog et al. 2009; Guillochon \& Ramirez-Ruiz 2013; Stone et al. 2013; Shiokawa et al. 2015; Bonnerot et al. 2016; Jiang et al. 2016b; Sạdowski et al. 2016). Despite significant progress in recent works, studying the detailed post-disruption dynamics is still challenging due to the three-dimensional nature, the complexity of the physics (general relativity, self-gravity, radiation, and magnetohydrodynamics), and the wide range of time/length scales involved (see Stone et al. 2018 for a review). An easier and more robust way of understanding the physics of TDEs is to look at the global properties such as mass, energy, and angular momentum through their conservation laws. In the simplest picture, if half of the star's mass is eventually accreted onto the $\mathrm{BH}$, the expected energy released is $\sim 10^{52}-10^{53} \mathrm{erg}$. However, for the majority of the TDE candidates found in recent surveys, the observed radiation energy in the optical/ UV bands is only $\sim 10^{51} \mathrm{erg}$. The puzzle is: where is the missing $90 \%-99 \%$ of the energy?

This low apparent radiative efficiency led Piran et al. (2015b) to suggest that the optical emission is powered by the shocks due to stream-stream collision near the apocenter of the elliptical orbits instead of the accretion flow near the BH. This is because the amount of kinetic energy dissipated at the shocks is $\sim 10^{51}\left(r_{\mathrm{I}} / 10^{3} r_{\mathrm{g}}\right)^{-1} \mathrm{erg}$, where $r_{\mathrm{I}}$ is the intersecting radius and $r_{\mathrm{g}} \equiv G M / c^{2}$ is the gravitational radius of the $\mathrm{BH}$. 
However, Piran et al. (2015b) did not consider $^{3}$ the radiation from the subsequent accretion onto the $\mathrm{BH}$ after the streamstream collision, which we argue should dominate the energy output from the TDE. This is because the shocks can only unbind less than half of the gas in the fallback stream due to the energy requirement. If part of the gas receives more energy and becomes unbound, the others become more tightly bound.

Thus, the majority of the shocked gas must be injected into the accretion flow, and the mass being fed to the accretion flow is a large fraction of that entering the self-intersecting shocks. The question of the low apparent radiative efficiency of the accretion flow is still unanswered. There are a number of possible solutions to this puzzle:

(1) The mass-feeding rate to the accretion flow may be highly super-Eddington at early time, and due to photon trapping and/or mass outflow, the luminosity of the escaping radiation may be regulated at a near-Eddington level. This radiatively inefficient accretion flow model is widely adopted by many authors (Loeb \& Ulmer 1997; Ulmer 1999; Strubbe \& Quataert 2009; Krolik \& Piran 2012; Coughlin \& Begelman 2014; Piran et al. 2015a; Metzger \& Stone 2016).

(2) It has been proposed by Svirski et al. (2017) that the accretion flow may be in the form of a highly eccentric disk and the angular momentum exchange among different parts of the disk may allow the majority ( $\gtrsim 90 \%$ ) of the mass to fall directly into the event horizon without circularization and viscous accretion.

(3) Most of the energy released from the accretion flow may be in the form of radiation either absorbed along the line of sight (e.g., by dust in the host galaxy) or at other wavelengths that have not yet been observed.

(4) Most of the energy released from TDEs may be in the form of relativistic jets beamed away from the Earth.

We discuss the first scenario in Sections 2 and 3. Based on the radiatively inefficient accretion flow model, we calculate the minimum bolometric energy output from a TDE and show that it is higher than the observed energy in the optical/nearUV bands by a factor of 10-100. Thus, this widely adopted model does not solve the energy efficiency puzzle.

The second scenario requires that (i) the disk eccentricity stays high for $\sim 10$ orbits $(\sim 1 \mathrm{yr})$ and (ii) the exchange of angular momentum among the fluid elements occurs in a way such that only $\lesssim 10 \%$ of the mass in the accretion flow circularizes and that the rest plunges into the event horizon of the BH (Svirski et al. 2017). Typically, the stream-stream collision occurs at a substantial angle $\left(40^{\circ}-160^{\circ}\right.$; Dai et al. 2015) and dissipates a large fraction of the orbital kinetic energy. In addition, the cooling time is generally comparable to or longer than the orbital time, so the resulting accretion flow is quasi-spherical (Hayasaki et al. 2016; Sa̧dowski et al. 2016) instead of highly elliptical. This scenario is not discussed in this paper, because it can only occur in rare cases where the streamstream collision angle is small ( $\ll 1 \mathrm{rad})$ and the dissipation of orbital energy is inefficient.

\footnotetext{
3 Another potential issue is that the shock-dissipated energy may not be radiated away efficiently. In order for the radiation to escape, the shocked gas needs to expand to a much larger volume than that occupied by the cold streams, and the radiation energy may be lost in the form of $P d V$ work. The stream-stream collision simulations by Jiang et al. (2016b) show that only a few percent of the initial kinetic energy is radiated away.
}

The third scenario may explain the radiative efficiency puzzle, considering that the optical/near-UV spectrum of TDEs is usually Rayleigh-Jeans-like and that the peak may be in the extreme-UV (EUV) band. This scenario has not been thoroughly explored in the literature, because it is difficult to infer the peak frequency and hence bolometric luminosity from the optical/near-UV data, considering the strong dependence on the (highly uncertain) line-of-sight extinction of the host galaxy (e.g., Gezari et al. 2009; Chornock et al. 2014). In Section 4, we show that this scenario is supported by existing dust reverberation observations in the mid-infrared where the extinction is negligible.

The fourth scenario, which involves relativistic off-axis jets, will be discussed in Section 5. We show that this scenario is ruled out by radio observations of ASASSN-14li (Holoien et al. 2014; Alexander et al. 2016; van Velzen et al. 2016a), but it still remains viable for the other thermal TDEs with only sparse radio upper limits.

In Section 6, we briefly discuss the implications of including the missing energy on the physics of circularization and accretion processes in TDEs. Throughout the paper, the convention of $Q=10^{n} Q_{n}$ and cgs units are used.

\section{Mass-feeding Rate to the Accretion Flow}

We consider a star of mass $M_{*}=m_{*} M_{\odot}$ and radius $R_{*}=r_{*} R_{\odot}$ interacting with a $\mathrm{BH}$ of mass $M=10^{6} M_{6} M_{\odot}$. The tidal disruption radius is expressed in units of the BH's gravitational radius $r_{\mathrm{g}} \equiv G M / c^{2}$ as

$$
\frac{r_{\mathrm{T}}}{r_{\mathrm{g}}} \simeq \frac{R_{*}}{r_{\mathrm{g}}}\left(\frac{M}{M_{*}}\right)^{1 / 3} \simeq 47 M_{6}^{-2 / 3} m_{*}^{-1 / 3} r_{*} .
$$

When the star first enters the radius $r_{\mathrm{T}}$, the tidal gravity of the $\mathrm{BH}$ causes a spread of specific energy across the star $-\Delta \epsilon \leqslant$ $\epsilon \leqslant \Delta \epsilon$ and (Stone et al. 2013)

$$
\Delta \epsilon=\eta_{\epsilon} \frac{G M R_{*}}{r_{\mathrm{T}}^{2}} \simeq 2.1 \times 10^{-4} c^{2} \eta_{\epsilon} M_{6}^{1 / 3} m_{*}^{2 / 3} r_{*}^{-1},
$$

where $\eta_{\epsilon}$ is a dimensionless number of order unity (depending on the stellar density profile and the detailed disruption process). Bound materials have negative specific energies $-\Delta \epsilon \leqslant \epsilon<0$ and the leading edge $(\epsilon=-\Delta \epsilon)$ has the minimum orbital period

$$
P_{\min } \simeq(41 \text { days }) \eta_{\epsilon}^{-3 / 2} M_{6}^{1 / 2} m_{*}^{-1} r_{*}^{3 / 2} .
$$

As shown in Figure 1, the bound stream self-intersects due to apsidal ${ }^{4}$ precession. Since the intersecting radius $r_{\mathrm{I}}$ is typically less than the apocenter radius of the most bound orbit, the time it takes for a fluid element to move from the beginning of the second orbit to the intersecting point is insensitive to the specific energy. Thus, the mass-flow rate to the intersecting

\footnotetext{
4 We ignore the Lense-Thirring precession arising from the BH's spin, which may otherwise deflect the stream out of the initial orbital plane. If the stream has a small enough cross section (the evolution of which is controlled by the competition among tidal shear, self-gravity, gas and magnetic pressure, etc.; see Kochanek 1994; Coughlin et al. 2016), such a deflection may prevent stream-stream intersection during the second orbit and hence circularization can be significantly delayed (Guillochon \& Ramirez-Ruiz 2015). As shown in Section 3, our conclusion on the radiation energy discrepancy will be even stronger because the mass-feeding rate to the disk may be reduced and the radiative efficiency of the accretion flow should be closer to that of the standard thin disk (Shakura \& Sunyaev 1973).
} 


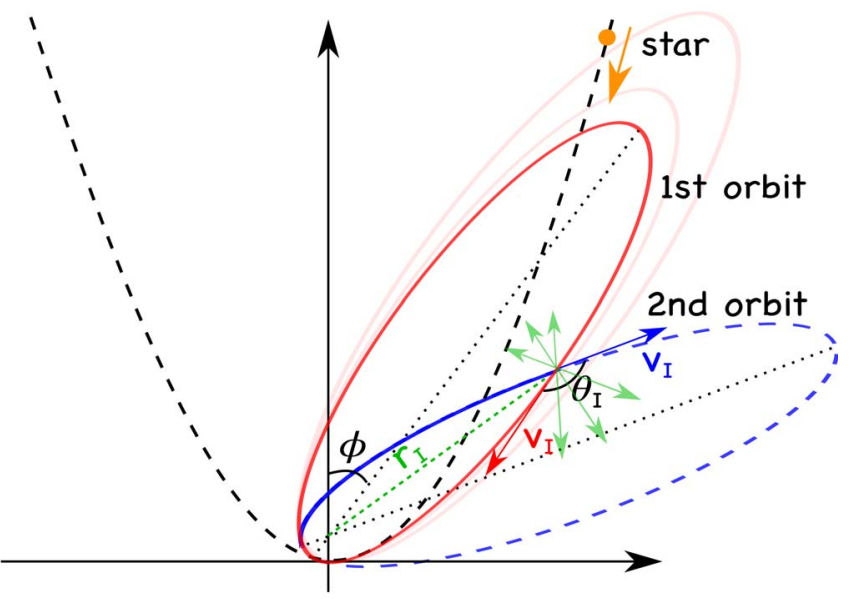

Figure 1. A schematic picture of a tidal disruption event. The star was initially in a parabolic orbit (dashed black curve). After the tidal disruption, the bound materials are in highly eccentric elliptical orbits of different semimajor axes (the most bound orbit is shown in red) but have the same apsidal precession angle $\phi$ per orbital cycle. Materials in their second orbit collide with those still in the first orbit with velocity $v_{\mathrm{I}}$ at radius $r_{\mathrm{I}}$ from the $\mathrm{BH}$, and the intersecting angle is $\theta_{\mathrm{I}}$. Then, the post-shock gas expands rapidly into a thick torus wrapping around the $\mathrm{BH}$.

point is given by

$$
\dot{M} \simeq \frac{d M}{d P}=\frac{d M}{d \epsilon} \frac{d \epsilon}{d P}=\frac{d M}{d \epsilon} \frac{(2 \pi G M)^{2 / 3}}{3} P^{-5 / 3},
$$

which depends on the (uncertain) specific energy distribution $d M / d \epsilon$.

In the following, we consider two simple models of specific energy distribution: (i) in the first ("frozen") case (e.g., Lodato et al. 2009; Stone et al. 2013; Coughlin et al. 2016), the distribution of specific energy is assumed to be "frozen" when the star first enters the tidal radius $r_{\mathrm{T}}$ and each fluid element evolves ballistically (ignoring self-gravity, internal pressure, and shocks), and (ii) in the second ("flat") case (e.g., Evans \& Kochanek 1989; Phinney 1989), $d M / d \epsilon$ is assumed to be independent of $\epsilon$ in the range $-\Delta \epsilon<\epsilon<\Delta \epsilon$, i.e.,

$$
\frac{d M}{d \epsilon}=\frac{M_{*}}{2 \Delta \epsilon}=2.3 \times 10^{3} \frac{M_{\odot}}{c^{2}} \eta_{\epsilon}^{-1} M_{6}^{-1 / 3} m_{*}^{1 / 3} r_{*} .
$$

We note that the specific energy distributions given by Newtonian and relativistic numerical simulations generally lie between these two extreme cases (e.g., Guillochon \& RamirezRuiz 2013; Cheng \& Bogdanović 2014; Coughlin \& Nixon 2015; Tejeda et al. 2017). We also define $t=0$ to be when the most tightly bound material (with $\epsilon=-\Delta \epsilon$ ) reaches the intersecting point.

Since the self-intersecting shock can only unbind a small fraction of the gas in the fallback stream, the mass enters the accretion flow at a rate roughly equal to that entering the shock. ${ }^{5}$ In the "frozen" case, when the star first enters the tidal radius $r_{\mathrm{T}}$, we assume that its spherical shape and original density profile are preserved and that all fluid elements are moving at the same velocity $v_{0}=\sqrt{2 G M / r_{\mathrm{T}}}$. Then, the specific orbital energy of each fluid element is assumed to be

\footnotetext{
5 Alternatively, one can assume that a fraction $f_{\mathrm{b}} \lesssim 1$ of the fallback gas joins the accretion flow. This factor can be easily included by replacing the thin-disk efficiency $\eta$ (see Equation (8) for the definition) by $f_{\mathrm{b}} \eta$ throughout this section, and our conclusion remains unchanged.
}

frozen until the stream self-intersects. For a polytropic equation of state $P \propto \rho^{\gamma}$, we solve the Lane-Emden equation for the density profile given by the total stellar mass $M_{*}$ and radius $R_{*}$. We consider three different polytropic indexes $\gamma=4 / 3,1.4,5 / 3$ (the star is more centrally concentrated for lower $\gamma$ ).

In the "flat" case, the mass-feeding rate to the accretion flow is

$$
\dot{M}(t) \simeq 3.0 M_{\odot} \mathrm{yr}^{-1} \frac{\eta_{\epsilon}^{3 / 2} m_{*}^{2}}{M_{6}^{1 / 2} r_{*}^{3 / 2}}\left(\frac{t+P_{\min }}{P_{\min }}\right)^{-5 / 3},
$$

which shows the canonical $t^{-5 / 3}$ behavior at $t \gg P_{\min }$ :

$$
\dot{M}(t) \simeq 7.8 \times 10^{-2} M_{\odot} \mathrm{yr}^{-1} \frac{M_{6}^{1 / 3} m_{*}^{1 / 3} r_{*}}{\eta_{\epsilon}}\left(\frac{t}{\text { year }}\right)^{-5 / 3}
$$

We note that the fallback rates calculated via grid (Guillochon \& Ramirez-Ruiz 2013) or smoothed-particle hydrodynamical (Coughlin \& Nixon 2015) simulations for $\left\{M_{6}=1, m_{*}=1\right.$, $\left.r_{*}=1, \gamma=5 / 3\right\}$ are quite similar with $\dot{M}(t=1$ year $) \simeq 9 \times$ $10^{-2} M_{\odot} \mathrm{yr}^{-1}$, which is close to our "flat" case if $\eta_{\epsilon} \simeq 0.9$.

In Figure 2, we show the distribution of the specific energy (left panel) and mass fallback rates (right panel) for the "frozen" and "flat" cases. We use $M_{*}=0.4 M_{\odot}, R_{*}=0.48 R_{\odot}$, and $M=$ $10^{6} M_{\odot}$. At sufficiently late time $t \gg P_{\min }$, the mass-feeding rate in all cases approaches the characteristic $t^{-5 / 3}$ power law because the specific energy distribution is asymptotically flat, $d M / d \epsilon \approx$ constant. The peak mass fallback rate is much smaller in the "frozen" cases, because more mass is concentrated near $\epsilon \approx 0$.

We define the Eddington accretion rate as

$$
\dot{M}_{\text {Edd }} \equiv \frac{L_{\text {Edd }}}{\eta c^{2}}=\left(2.6 \times 10^{-2} M_{\odot} \mathrm{yr}^{-1}\right) M_{6} \eta_{-1}^{-1},
$$

where $L_{\mathrm{Edd}}=1.5 \times 10^{44} M_{6} \mathrm{erg} \mathrm{s}^{-1}$ is the Eddington luminosity for solar metallicity with Thomson scattering opacity and $\eta=0.1 \eta_{-1}$ is the radiative efficiency ${ }^{6}$ for a standard thin disk (Shakura \& Sunyaev 1973). In the "flat" case, the ratio between the peak mass-feeding rate and the Eddington accretion rate is

$$
\frac{\dot{M}_{\text {peak }}}{\dot{M}_{\text {Edd }}} \simeq 114 \eta_{\epsilon}^{3 / 2} \eta_{-1} M_{6}^{-3 / 2} m_{*}^{2} r_{*}^{-3 / 2},
$$

which becomes less than unity for $\mathrm{BH}$ mass $M \gtrsim 2.3 \times$ $10^{7} M_{\odot} \eta_{\epsilon} \eta_{-1}^{2 / 3} m_{*}^{4 / 3} r_{*}^{-1}$. On the right panel of Figure 2, we mark the Eddington accretion rate for the (conservative) efficiency $\eta=0.05$ with a horizontal dashed line. In all the cases considered in Figure 2, the mass fallback rates exceed the Eddington accretion rate for $\sim 1 \mathrm{yr}$.

\section{Minimum Bolometric Energy}

Super-Eddington $\mathrm{BH}$ accretion flows $\left(\dot{M} \gg \dot{M}_{\mathrm{Edd}}\right)$ are generally believed to be radiatively inefficient compared to sub-Eddington thin disks, because (i) radiation can be trapped

\footnotetext{
6 Note that $\eta=1-\sqrt{1-2 r_{\mathrm{g}} /\left(3 r_{\mathrm{ISCO}}\right)}$ ranges from 0.038 (for a retrograde orbit around an extreme Kerr BH with spin -1) to 0.42 (for a prograde orbit around an extreme Kerr BH with spin 1), where $r_{\text {ISCO }}$ is the radius of the innermost stable circular orbit. For a zero-spin Schwarzschild $\mathrm{BH}$, we have $\eta=0.057$.
} 

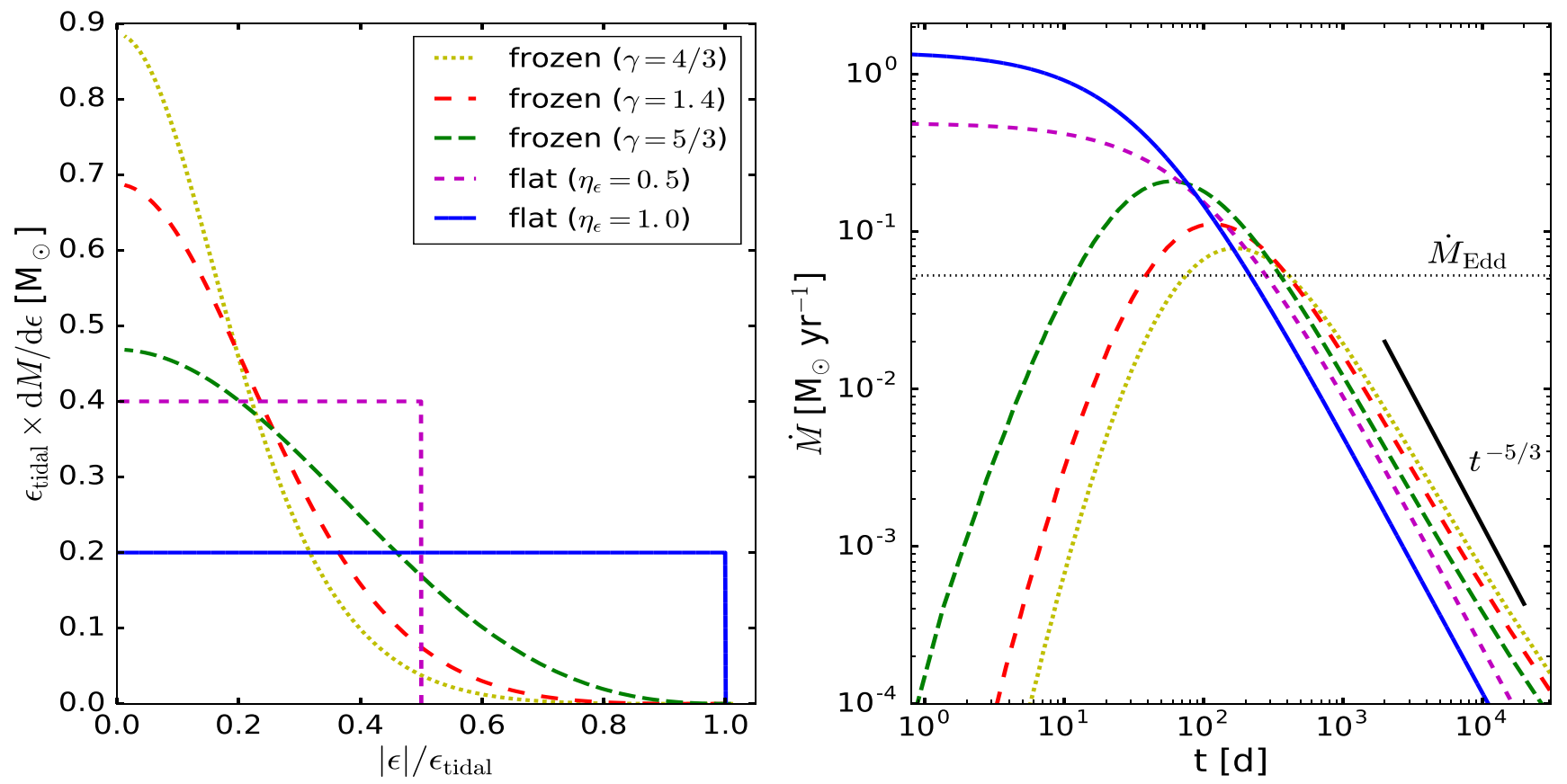

Figure 2. Left panel: the yellow, red, and green curves show the distributions of specific energy when the star first enters $r_{\mathrm{T}}$, under the assumption that it keeps its spherical shape and original density profile. We assume a polytropic equation of state and solve the Lane-Emden equation under different polytropic indexes $\gamma=4 / 3$, 1.4 , and $5 / 3$. The specific energy is normalized by the work per unit mass done by tidal forces $\epsilon_{\text {tidal }} \equiv G M R / r_{\mathrm{T}}^{2}$. The magenta and blue curves show a flat distribution of specific energy in $(-\Delta \epsilon, 0)$, where $\Delta \epsilon=\eta_{\epsilon} \epsilon_{\text {tidal }}$. We use BH mass $M=10^{6} M_{\odot}$, stellar mass $0.4 M_{\odot}$, and stellar radius $0.48 R_{\odot}$ for this figure. Right panel: mass fallback rate for the different cases in the left panel. We define $t=0$ to be when the most tightly bound material (with $\epsilon=-\Delta \epsilon$ ) reaches the intersection point. At late time, the fallback rate goes as $t^{-5 / 3}$ since $d M / d \epsilon$ is asymptotically flat. The horizontal dashed line shows the Eddington accretion rate $\dot{M}_{\text {Edd }}=5.3 \times 10^{-2} M_{\odot} \mathrm{yr}^{-1}$ according to Equation (8) with thin-disk radiation efficiency $\eta=0.05$.

by the inflowing matter and advected into the BH (Begelman 1979), and (ii) the majority of the accreting mass may become unbound due to energy injection along with the outward transport of angular momentum (Narayan \& Yi 1994; Blandford \& Begelman 1999, 2004). As a result, the bolometric radiative luminosity may be regulated at the Eddington luminosity times a logarithmic factor of order unity, as shown in recent multidimensional simulations of superEddington BH accretion disks (Ohsuga \& Mineshige 2011; McKinney et al. 2014; Sądowski \& Narayan 2016). We note that the vertical advection of radiation due to magnetic buoyancy may transport radiation energy faster than diffusion, which may allow the accretion disk to radiate at highly superEddington luminosities (e.g., Blaes et al. 2011; Jiang et al. 2014), although the radiation may suffer from adiabatic loss if the disk also launches a highly optically thick wind. Another possibility for higher radiative efficiency is that the accumulation of magnetic flux near the $\mathrm{BH}$ event horizon leads to a magnetically arrested disk (magnetic fields become dynamically important), where radiation escapes along the low-density channel created by the Poynting flux of rotating field lines (Narayan et al. 2003; McKinney et al. 2015).

In this section, we calculate the minimum bolometric energy from a TDE, according to the mass-feeding rates to the accretion flow obtained in the previous section. Our calculation is based on two conservative assumptions: (i) when the massfeeding rate to the accretion flow exceeds the Eddington accretion rate, the angle-integrated bolometric luminosity is at least $L_{\mathrm{Edd}}$, and (ii) when the mass-feeding rate drops below the Eddington accretion rate, the bolometric emission from the disk tracks the mass-feeding rate, and the luminosity becomes $\eta \dot{M} c^{2}$ as in the standard thin-disk model, i.e.,

$$
\begin{cases}L_{\mathrm{bol}}(t) \gtrsim L_{\mathrm{Edd}} & \text { if } \dot{M}(t) \geqslant \dot{M}_{\mathrm{Edd}} \\ L_{\mathrm{bol}}(t) \simeq \eta \dot{M} c^{2} & \text { if } \dot{M}(t)<\dot{M}_{\mathrm{Edd}}\end{cases}
$$

The total bolometric radiation energy is given by

$$
E_{\mathrm{bol}} \equiv \int_{0}^{\infty} L_{\mathrm{bol}}(t) d t
$$

Note that, if the accretion is significantly delayed (e.g., due to slow circularization and/or viscous spreading) with respect to the mass-injection rate given by Equation (4), the peak accretion rate is reduced, and the total bolometric radiation energy will be higher ${ }^{7}$ (since the radiation efficiency of the accretion flow is closer to that of a standard thin disk).

We also denote the duration of super-Eddington accretion as $t_{\text {Edd }}$ (and $t_{\text {Edd }}=0$ if $\dot{M}_{\text {peak }}<\dot{M}_{\text {Edd }}$ ). In the "flat" specific energy distribution case, if $\dot{M}_{\text {peak }} \gg \dot{M}_{\text {Edd }}, t_{\text {Edd }}$ can be solved from $\dot{M}\left(t_{\text {Edd }}\right)=\dot{M}_{\text {Edd }}$ (combining Equations (6) and (8)),

$$
t_{\text {Edd }} \simeq(700 \text { days }) \eta_{\epsilon}^{-3 / 5} \eta_{-1}^{3 / 5} M_{6}^{-2 / 5} m_{*}^{1 / 5} r_{*}^{3 / 5} .
$$

\footnotetext{
7 General relativistic hydrodynamical simulations by Shiokawa et al. (2015) showed that the circularization process after the initial stream-stream collision may take up to $\sim 10 P_{\min }(\sim 1 \mathrm{yr})$ and hence the accretion process may be significantly lengthened at a reduced accretion rate. The viscous evolution of the accretion flow with a time-dependent mass feeding has been studied by Cannizzo et al. (1990) and Shen \& Matzner (2014). TDEs with a prolonged accretion phase of $\sim 1 \mathrm{yr}$ and slow variability may have been missed in current optical/UV surveys (T. Holoien 2018, private communication).
} 

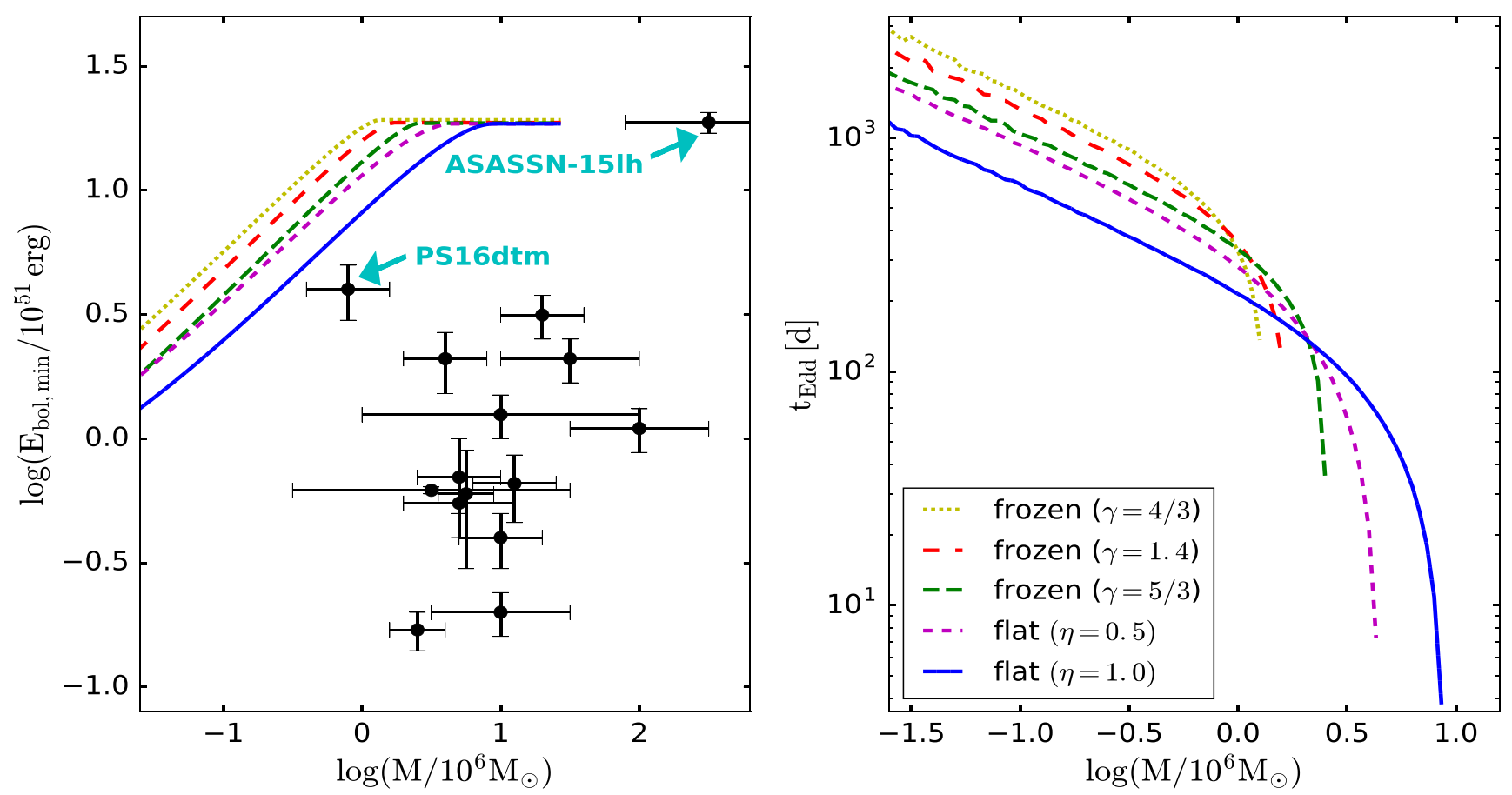

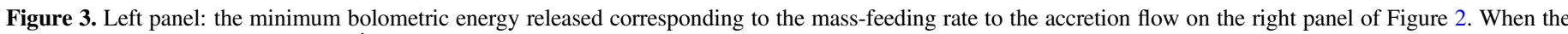

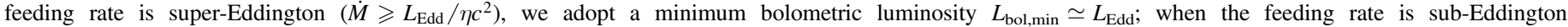

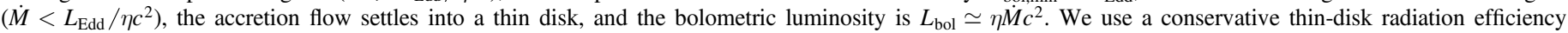

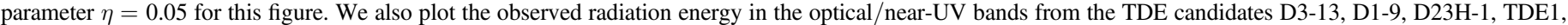

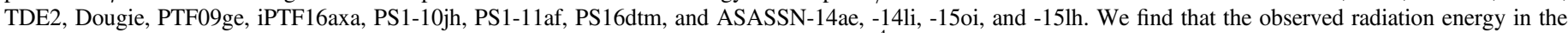

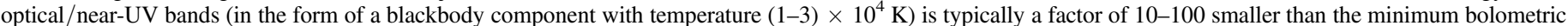

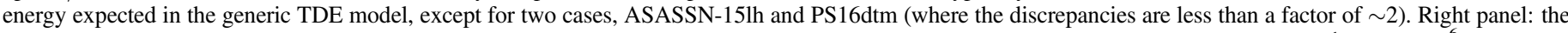

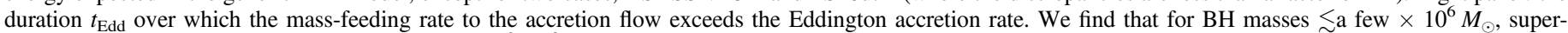
Eddington mass feeding lasts for $t_{\text {Edd }} \gtrsim$ a few $\times 10^{2}-10^{3}$ days.

Then, the minimum bolometric energy is

$$
\begin{aligned}
E_{\mathrm{bol}} & \gtrsim L_{\mathrm{Edd}} t_{\mathrm{Edd}}+\int_{t_{\mathrm{Edd}}}^{\infty} L_{\mathrm{Edd}}\left(\frac{t}{t_{\mathrm{Edd}}}\right)^{-5 / 3} d t \simeq \frac{5}{2} L_{\mathrm{Edd}} t_{\mathrm{Edd}} \\
& \simeq\left(2.3 \times 10^{52} \mathrm{erg}\right) \eta_{\epsilon}^{-3 / 5} \eta_{-1}^{3 / 5} M_{6}^{3 / 5} m_{*}^{1 / 5} r_{*}^{3 / 5}
\end{aligned}
$$

where we have used $\dot{M}(t) \propto t^{-5 / 3}$ when $t \gg P_{\min }$. Note that Equations (12) and (13) are only valid when $\dot{M}_{\text {peak }} \gg \dot{M}_{\text {Edd }}$, i.e., for $\mathrm{BH}$ masses $M \lesssim\left(1.1 \times 10^{7} M_{\odot}\right) \eta_{\epsilon} \eta_{-1}^{2 / 3} m_{*}^{4 / 3} r_{*}^{-1}$; for larger $\mathrm{BH}$ masses, the super-Eddington duration $t_{\mathrm{Edd}}$ quickly drops to zero, and the bolometric energy released $E_{\text {bol }}$ approaches $\eta M_{*} c^{2} / 2 \simeq\left(9 \times 10^{52} \mathrm{erg}\right) \eta_{-1} m_{*}$.

On the left panel of Figure 3, we show the minimum bolometric energy released corresponding to the mass-feeding rates shown on the right panel of Figure 2. We use the thin-disk radiative efficiency parameter $\eta=0.05$ for this figure. We also plot the observed radiation energy in the optical/near-UV bands from the TDE candidates found by GALEX (D3-13, D1-9, D23H-1; Gezari et al. 2006, 2008, 2009), SDSS (TDE1, TDE2; van Velzen et al. 2011), ROTSE (Dougie; Vinkó et al. 2015), PTF (PTF09ge, iPTF16axa; Arcavi et al. 2014; Hung et al. 2017a), Pan-STARRS (PS1-10jh, PS1-11af, PS16dtm; Gezari et al. 2012; Chornock et al. 2014; Blanchard et al. 2017), and ASASSN (-14ae, -14li, -15oi, -15lh, Holoien et al. 2014, 2016a, 2016b; Dong et al. 2016). Only Galactic extinction has been corrected for these events. Our sample is selected from the open TDE catalog (http://tde. space) based on the good quality of the optical/near-UV photometry in these sources. We find that the observed radiation energy in the optical/near-UV bands, typically in the form of a blackbody component with temperature $(1-3) \times 10^{4} \mathrm{~K}$, is a factor of 10-100 smaller than the minimum bolometric energy expected in the generic TDE model. This conclusion is not sensitive to the different fallback-rate models. We note that ASASSN-15lh and PS16dtm are the only two cases with observed radiation energies near the theoretical expectation to within a factor of $\sim 2$. It is still debated whether these two sources are TDEs or superluminous supernovae (see Metzger et al. 2015; Dong et al. 2016; Leloudas et al. 2016; Blanchard et al. 2017). We also note that the missing energy is not observed in the X-ray band $(0.2-10 \mathrm{keV})$ because the ratios between the X-ray and optical/near-UV luminosities for these TDE candidates are at most of order unity (Auchettl et al. 2017).

It can be seen from Equation (13) that this energy discrepancy cannot be reconciled by varying the thin-disk radiative efficiency $\eta \in(0.038,0.42)$, specific energy distribution parameter $\eta_{\epsilon} \in(\sim 0.3, \sim 1)$, stellar mass $m_{*} \gtrsim 0.1$ (for a main-sequence star), and stellar radius $r_{*} \gtrsim 0.2$.

We note that highly centrally concentrated stars (e.g., the Sun) are only fully disrupted for deeply penetrating orbits, while for the more general situation, the total fallback mass is smaller than $M_{*} / 2$ (Mainetti et al. 2017). However, it is unlikely that the majority of the observed TDEs are partial disruptions where the star only loses $\ll 0.1 M_{\odot}$ of mass, because the bright optical emission requires a minimum amount of mass to reprocess the EUV or soft X-ray emission from the accretion flow (Piran et al. 2015b; Metzger \& 
Stone 2016; Roth et al. 2016). In the following, we show that this minimum reprocessing layer constrains the total fallback mass to be more than $\sim 0.1 M_{\odot}$ for most TDEs in our sample.

The blackbody-like optical/near-UV spectrum of a TDE gives the emitting area

$$
A=L_{\mathrm{BB}} / \sigma T^{4} \simeq\left(1.8 \times 10^{30} \mathrm{~cm}^{2}\right) L_{\mathrm{BB}, 44} T_{4.5}^{-4},
$$
where $\sigma$ is the Stefan-Boltzmann constant, $L_{\mathrm{BB}}$ is the luminosity of the optical/near-UV component, and $T$ is the photospheric temperature. If the emitting surface covers a solid angle of $\Omega(\leqslant 4 \pi)$, the corresponding photospheric radius is

$$
r_{\mathrm{ph}} \simeq\left(3.7 \times 10^{14} \mathrm{~cm}\right) L_{\mathrm{BB}, 44}^{1 / 2} T_{4.5}^{-2} \sqrt{4 \pi / \Omega} .
$$

The location of the photosphere is marked by the effective absorption optical depth $\tau \simeq \rho \kappa r_{\mathrm{ph}} \simeq 1$, where the effective opacity is given by $\kappa=\sqrt{\kappa_{\mathrm{abs}} \kappa_{\mathrm{s}}}$ in the scattering-dominated regime, and $\kappa_{\mathrm{abs}}$ and $\kappa_{\mathrm{s}}$ are the opacities for absorption and electron scattering respectively. Thus, the mass density near the photosphere is given by

$$
\rho \simeq\left(\kappa r_{\mathrm{ph}}\right)^{-1} \simeq\left(10^{-13.5} \mathrm{~g} \mathrm{~cm}^{-3}\right) \kappa_{-1}^{-1} r_{\mathrm{ph}, 14.5}^{-1},
$$

where we have used a fiducial effective opacity of $\kappa=0.1 \kappa_{-1} \mathrm{~cm}^{2} \mathrm{~g}^{-1}$ motivated by the fact that bound-free and free-free opacity $\kappa_{\mathrm{abs}} \ll \kappa_{\mathrm{s}} \simeq 0.34 \mathrm{~cm}^{2} \mathrm{~g}^{-1}$ (for solar abundance) at densities $\rho \lesssim 10^{-13} \mathrm{~g} \mathrm{~cm}^{-3}$ and temperatures $T \gtrsim 10^{4} \mathrm{~K}$ (a rough scaling is $\kappa_{\text {abs }} \propto \rho T^{-3.5}$ ). The true opacity depends on gas metallicity and the detailed ionization state near the photosphere (see a discussion by Roth et al. 2016).

On the other hand, since the fallback materials do not have enough angular momentum to circularize at radii $r_{\mathrm{ph}} \gtrsim 10^{14} \mathrm{~cm}$, the widths of the ( $\mathrm{H} \alpha$ and/or He II) emission lines cannot be sufficiently broadened by gas rotation. Instead, they suggest that the gas in the line-forming region near the photosphere is expanding outwards at typical speeds $v=10^{9} v_{9} \mathrm{~cm} \mathrm{~s}^{-1}$. To sustain the bright optical emission for a duration of $\Delta t$ (typically $\sim 30$ days), the reprocessing gas needs to have a minimum mass 8

$$
M_{\text {rep }} \gtrsim \rho A v \Delta t \simeq\left(0.06 M_{\odot}\right) \frac{L_{\mathrm{BB}, 44}^{1 / 2}}{T_{4.5}^{2} \kappa_{-1}} \sqrt{\frac{\Omega}{4 \pi}} \frac{v_{9} \Delta t}{30 \text { days }} .
$$

To launch this outflow through the local dissipation of orbital energy (e.g., via stream-stream collision as shown in Jiang et al. 2014), the total amount of fallback (bound) mass needed is $M_{\mathrm{b}} \gtrsim 2 M_{\text {rep }} \gtrsim 0.1 M_{\odot}$.

Another possible solution to the energy discrepancy is that the BH masses in these TDEs are actually $\lesssim 10^{5} M_{\odot}$. For some of the TDEs in our sample, BH masses lower (by up to a factor of 10) than the values reported in the discovery papers were indeed found by Wevers et al. (2017) from the velocity dispersion of the host galaxies using high-resolution spectroscopy. On the right panel of Figure 3 , we show the duration $t_{\text {Edd }}$ over which the mass-feeding rate to the accretion flow exceeds the Eddington accretion rate. We can see that, if the luminosity

\footnotetext{
A speculative "TDE impostor" idea is the sudden accretion of $\sim 0.01 M_{\odot}$ following stellar collisions due to consecutive extreme mass ratio inspirals (EMRIs, Metzger \& Stone 2017). In such cases, the peak accretion luminosity can reach $\sim 10^{44} \mathrm{erg} \mathrm{s}^{-1}$ but mainly in the EUV or soft X-ray bands. To match the observed optical/near-UV luminosity of $\sim 10^{44} \mathrm{erg} \mathrm{s}^{-1}$, this model relies on the reprocessing by a fossil disk (with mass $\sim 0.1 M_{\odot}$ ) at distances $10-100$ au from the BH. The disk must exist for $\gtrsim 10^{4} \mathrm{yr}$ to witness the stellar collision and hence is geometrically thin. It is unclear whether such a thin disk is capable of reprocessing a large fraction (order unity) of the EUV/soft X-ray emission from the TDE impostor.
}

is indeed capped near the Eddington luminosity of $\lesssim 10^{5} M_{\odot}$ $\mathrm{BHs}$, then the radiation energy should be released on a timescale of $\gtrsim 10^{3}$ days (Equation (12)), which is inconsistent with the observed rise and decay time in the TDEs in our sample. Additionally, many TDE candidates had peak optical/ near-UV luminosities of order $10^{44} \mathrm{erg} \mathrm{s}^{-1}$, which is much higher than $L_{\mathrm{Edd}}$ for $\mathrm{BH}$ masses $\lesssim 10^{5} M_{\odot}$.

\section{The Extreme-UV Scenario}

In the previous section, we show that 10-100 times the observed energy may have escaped our detection. For the TDEs in our sample, the optical/near-UV spectrum is usually Rayleigh-Jeans-like, so most of the missing energy may be in the EUV band (there are stringent upper limits in the X-ray band). This scenario is also motivated by the temperature associated with an Eddington luminosity emerging at the tidal disruption radius (Ulmer 1999),

$$
k T \simeq k\left(\frac{L_{\mathrm{Edd}}}{4 \pi r_{\mathrm{T}}^{2} \sigma_{\mathrm{SB}}}\right)^{1 / 4} \sim(20 \mathrm{eV}) M_{6}^{1 / 12} r_{*}^{-1 / 2} m_{*}^{1 / 6},
$$

where $k$ is the Boltzmann constant and $\sigma_{\mathrm{SB}}$ is the StefanBoltzmann constant. This EUV emission may be directly observable for high-redshift TDEs, provided that photons near the spectral peak do not suffer from strong absorption (by, e.g., neutral hydrogen) in the host galaxy.

In this section, we discuss another indirect way of constraining the total UV-optical luminosity with dust reverberation mapping in the mid-infrared. If the gaseous medium at the galactic center is dusty, a fraction of the UV photons will be absorbed by dust and re-radiated in the midinfrared wavelengths $\lambda \sim 3-10 \mu \mathrm{m}$ (Lu et al. 2016). In the optically thin limit, due to high UV fluxes, dust particles will sublime within the critical radius (Waxman \& Draine 2000),

$$
R_{\mathrm{sub}} \sim(0.2 \mathrm{pc}) L_{\mathrm{UV}, 45}^{1 / 2}\left(T_{\mathrm{sub}} / 1800 \mathrm{~K}\right)^{-2.5} a_{-5}^{-1 / 2},
$$

where $L_{\mathrm{UV}}=10^{45} L_{\mathrm{UV}, 45} \mathrm{erg} \mathrm{s}^{-1}$ is the total UV-optical luminosity of the TDE, $T_{\text {sub }}$ is the sublimation temperature, and $a=0.1 a_{-5} \mu \mathrm{m}$ is the grain radius. The mid-infrared emission from the surviving dust particles at radius $R \sim R_{\text {sub }}$ lasts for a light-crossing timescale of the radiating dust shell

$$
t_{\mathrm{IR}} \sim \frac{R_{\mathrm{sub}}}{c} \simeq(0.6 \text { year }) L_{\mathrm{UV}, 45}^{1 / 2}\left(\frac{T_{\mathrm{sub}}}{1800 \mathrm{~K}}\right)^{-2.5} a_{-5}^{-1 / 2} .
$$

Therefore, we can monitor the mid-infrared emission from a sample of newly discovered TDEs on a cadence of a few months and then use the measured duration $t_{\mathrm{IR}}$ to infer the total UV-optical luminosity $L_{\mathrm{UV}}$.

We note that Equations (19) and (20) only capture the qualitative behavior of dust reverberation mapping. More detailed calculations are needed to extract the total UV-optical luminosity from mid-infrared observations. The grain temperature at a certain distance from the UV source depends on the infrared emissivity, which is uncertain at high temperatures $\gtrsim 1000 \mathrm{~K}$ (Draine \& Lee 1984). The sublimation temperature $T_{\text {sub }}$ depends on the size (distribution) and composition of dust particles (Guhathakurta \& Draine 1989). More importantly, the mid-infrared duration $t_{\mathrm{IR}}$ depends on the spatial distribution of dust near the galactic center as well as on the observational wavelength (the emission spectrum from grains of a certain temperature is not a blackbody and colder dust particles emit at 
longer wavelengths). For instance, different dust distribution geometries (e.g., torus, patchy clouds) could cause $t_{\mathrm{IR}}$ to vary by a factor of a few, so the UV-optical luminosity may have an uncertainty of an order of magnitude $\left(L_{\mathrm{UV}} \propto t_{\mathrm{IR}}^{2}\right)$. More detailed calculations taking these uncertain factors into account are left for future works.

Currently, a few TDEs have been observed by the Wide-field Infrared Survey Explorer at 3.6 and $4.5 \mu \mathrm{m}$ (Jiang et al. 2016a; van Velzen et al. 2016b; Dou et al. 2017). PTF09ge had a midinfrared luminosity of $L_{\mathrm{IR}} \sim 10^{42} \mathrm{erg} \mathrm{s}^{-1}$ lasting for $t_{\mathrm{IR}} \sim 1 \mathrm{yr}$ (van Velzen et al. 2016b). This implies an EUV luminosity of $L_{\mathrm{UV}} \sim 10^{45} \mathrm{erg} \mathrm{s}^{-1}$ (but note the large uncertainties mentioned above), which is a factor of $\sim 10$ higher than the optical/nearUV luminosity. If the duration of the EUV emission equals that of the optical emission, then the total energy released from PTF09ge may be of order $10^{52} \mathrm{erg}$. Future multiwavelength mid-infrared observations by JWST will enable detailed modeling of the dust properties/spatial distribution, and hence we can measure the total UV-optical energy released from TDEs more accurately. This method of reverberation mapping, commonly used in the AGN community (e.g., Peterson 1993), can be used to constrain the total energy reservoir of TDEs and may provide a solution to the energy efficiency puzzle.

\section{The Jet Scenario}

In this section, we discuss the possibility that $10^{52}-10^{53} \mathrm{erg}$ of energy is carried away by narrowly beamed relativistic jets. This is motivated by the powerful nonthermal $\gamma$-/X-ray emission from Swift J1644+57 (Bloom et al. 2011; Burrows et al. 2011), Swift J2058+05 (Cenko et al. 2012), and perhaps a third one, Swift J1112+82 (Brown et al. 2015). The isotropic equivalent energies released in the X-ray band in Swift $\mathrm{J} 1644+57$ and $\mathrm{J} 2058+05$ are $E_{X}^{\text {(iso) }} \simeq 5 \times 10^{53}$ erg and $9 \times$ $10^{53} \mathrm{erg}$, respectively. The total energy carried by the jet is given by $E_{\mathrm{j}}=E_{\mathrm{X}} f_{\mathrm{b}, \mathrm{X}} f_{\mathrm{bol}}^{-1} f_{\mathrm{r}}^{-1}$, where $f_{\mathrm{b}, \mathrm{X}}$ is the beaming factor of the X-ray emission, $f_{\text {bol }}^{-1}$ is the bolometric correction (to account for $\gamma$-rays), and $f_{\mathrm{r}}$ is the jet radiative efficiency. Despite large uncertainties in these factors, we can see that the jet may indeed carry up to $\sim 10^{53}$ erg of energy in these cases (e.g., if $f_{\mathrm{b}, \mathrm{X}} \sim 0.02$ and $f_{\mathrm{bol}}^{-1} f_{\mathrm{r}}^{-1} \sim 10$ for Swift $\mathrm{J} 1644+57$ ).

There are a number of arguments against this jet scenario for the majority of TDEs. First, only about $10 \%$ of AGNs are radio loud with relativistic jets (e.g., Wilson \& Colbert 1995), but this argument may be weak if the properties of the TDE accretion flow differ qualitatively from those of AGNs. We also note that the jet power in radio-loud AGNs is on the same order as the disk luminosity (e.g., Ghisellini et al. 2014), so the EUV scenario and the jet scenario may operate together.

Second, the rate of events like Swift J1644 +57 is estimated to be $\sim 10^{-9}$ galaxy $^{-1} \mathrm{yr}^{-1}$, if one assumes that the Swift BAT is sensitive to all events within its field of view $(\sim 4 \pi / 7)$ in the past $\sim 10$ years up to redshift $\sim 1$ (Burrows et al. 2011; Cenko et al. 2012; Brown et al. 2015). This extremely small rate is four orders of magnitude lower than the observationally inferred TDE rate of $\sim 10^{-5}$ galaxy $^{-1} \mathrm{yr}^{-1}$, and it requires a combination of a small beaming factor and that only a small fraction of TDEs produce relativistic jets. However, the fact that all three jetted TDEs were discovered within three months of the same year raises the question of incompleteness. Additionally, relativistic jets from TDEs may release the majority of their radiation in the X-ray band, and they do not trigger Swift BAT in the $\gamma$-ray band. Thus, the observed nonthermal TDEs may be tip-of-an-iceberg cases of a much larger population of jetted events, so this argument is also a weak one.

Third, powerful radio emission as seen in Swift J1644+57 is expected to be observable even for off-axis observers at sufficiently late times $t \gtrsim 1 \mathrm{yr}$ when the jet decelerates to nonrelativistic speeds (Giannios \& Metzger 2011). However, the flux at a given frequency seen by an off-axis observer depends sensitively on the poorly known circumnuclear medium (CNM) density profile, the fraction of energy in magnetic fields in the shocked region $\left(\epsilon_{\mathrm{B}}\right)$, and the angular structure of the jet (Sari et al. 1998; Kumar \& Granot 2003). Many of the thermal TDEs in our sample only had (typically single-epoch) upper limits ${ }^{9}$ at $5 \mathrm{GHz}$ at $\sim 1-10 \mathrm{yr}$ after the optical peak (van Velzen et al. 2013). Generozov et al. (2017) explored a wide range of CNM densities and used these radio upper limits to constrain the jet energies to be $E_{\mathrm{j}} \lesssim 10^{52}-10^{53}$ erg (mostly near the higher end; see their Table 2). In their modeling, they kept fixed the magnetic energy fraction $\left(\epsilon_{\mathrm{B}}=0.002\right)$ and the angular structure of the jet (a fast narrow core with Lorentz factor 10 surrounded by a slow wide sheath with Lorentz factor 2, as in Mimica et al. 2015). Including the uncertainties in these two aspects (especially lower $\epsilon_{\mathrm{B}}$ ) may make their constraints even weaker.

The most constraining case is ASASSN-14li (luminosity distance $D_{\mathrm{L}} \approx 90 \mathrm{Mpc}$ ) where the radio luminosity is many orders of magnitude lower and has decayed rapidly since the first detection (at $\sim 1$ month after the optical peak; van Velzen et al. 2016a). In the following, we show that a powerful jet with energy $E_{\mathrm{j}} \sim 10^{52}-10^{53} \mathrm{erg}$ is inconsistent with multiwavelength observations from 1.45 to $24.5 \mathrm{GHz}$ at late time $t \gtrsim 150$ days (Alexander et al. 2016).

At sufficiently late times, the shock front expands in a nearly spherical manner at a nonrelativistic speed $\beta c$ and radius $r \simeq \beta c t$ from the $\mathrm{BH}$. We assume the CNM density profile $n(r)$ to be sufficiently smooth (e.g., a power-law function), so the total number of protons (or electrons) in the shocked region is

$$
N_{\mathrm{tot}} \simeq \frac{E_{\mathrm{j}}}{m_{\mathrm{p}} \beta^{2} c^{2} / 2} \simeq \frac{4 \pi}{3}(\beta c t)^{3} n,
$$

which gives $n \simeq\left(54 \mathrm{~cm}^{-3}\right) E_{\mathrm{j}, 52} \beta^{-5}(t / 150 \text { days })^{-3}$ at radius $r$. The magnetic field strength in the shocked region is

$$
B \simeq\left(16 \pi \epsilon_{\mathrm{B}} n m_{\mathrm{p}} \beta^{2} c^{2}\right)^{1 / 2} \simeq\left(2.7 \times 10^{-3} \mathrm{G}\right) n^{1 / 2} \beta \epsilon_{\mathrm{B},-4}^{1 / 2} .
$$

The spectral peak is at the synchrotron self-absorption frequency $\nu_{\mathrm{a}}$, at which the flux density $F_{\nu_{\mathrm{a}}}$ is given by

$$
4 \pi D_{\mathrm{L}}^{2} F_{\nu_{\mathrm{a}}} \simeq \frac{2 \nu_{\mathrm{a}}^{2}}{c^{2}} \gamma_{\mathrm{a}} m_{\mathrm{e}} c^{2} 4 \pi^{2} r^{2},
$$

where $\gamma_{\mathrm{a}}=\left(4 \pi m_{\mathrm{e}} c \nu_{\mathrm{a}} / 3 e B\right)^{1 / 2}$ is the electron Lorentz factor corresponding to synchrotron frequency $\nu_{\mathrm{a}}$. We combine Equations (21)-(23) and obtain

$$
F_{\nu_{\mathrm{a}}} \simeq(125 \mathrm{mJy}) \beta^{\frac{11}{4}} \nu_{\mathrm{a}, 9}^{\frac{5}{2}}(t / 150 \text { days })^{\frac{11}{4}} E_{\mathrm{j}, 52}^{-\frac{1}{4}} \epsilon_{\mathrm{B},-4}^{-\frac{1}{4}} .
$$

Therefore, in the off-axis jet scenario, the shock expansion speed can be calculated from the three measurables $\nu_{\mathrm{a}}, F_{\nu_{\mathrm{a}}}$,

\footnotetext{
9 Similar upper limits have been reported by Bower et al. (2013) for a sample of X-ray-selected TDE candidates.
} 
and $t$ by

$$
\beta \simeq 0.17\left(F_{\nu_{\mathrm{a}}} / \mathrm{mJy}\right) \frac{4}{11} \nu_{\mathrm{a}, 9}^{-\frac{11}{10}}(t / 150 \text { days })^{-1} E_{\mathrm{j}, 52}^{\frac{1}{11}} \epsilon_{\mathrm{B},-4}^{\frac{1}{11}} .
$$

The peak frequency $\nu_{\mathrm{a}}$ and flux density $F_{\nu_{\mathrm{a}}}$ at $t=(143,207,246$, $304,381)$ days measured by Alexander et al. (2016) give expansion speeds $\beta \simeq(2.2,2.6,2.4,2.9,2.8) \times 10^{-2}\left(E_{\mathrm{j}, 52} \epsilon_{\mathrm{B},-4}\right)^{1 / 11} . \mathrm{A}$ similar conclusion of a nearly constant expansion speed is drawn by Krolik et al. (2016), under the assumption that the magnetic field and electrons are in energy equipartition (although we do not make this assumption). This nearly free expansion of the "synchrotron photosphere" at a subrelativistic speed seen in the radio band is inconsistent with the deceleration of a blast wave in the Sedov regime, and hence a relativistic off-axis jet scenario with $E_{\mathrm{j}} \sim 10^{52}-10^{53}$ erg is ruled out for ASASSN-14li.

We conclude that the jet scenario, where most of the energy released from the TDE accretion flow is in the form of narrowly beamed relativistic jets, is ruled out for ASASSN-14li, but it is still a viable solution to the energy efficiency puzzle for the other thermal TDEs. This is mostly because single-epoch upper limits are by far less constraining than multiwavelength and multiepoch detections.

\section{Discussion}

In previous sections, we have shown that $90 \%-99 \%$ of the energy released from the accretion flow is not detected for the majority of TDEs. In the following, we discuss the implications of the missing energy on the physics of circularization and accretion in TDEs.

We argue that, at least for some TDEs, the missing energy (in the EUV and/or in the form of relativistic jets) may be released within a few times the orbital period of the most bound material $P_{\min }$ (see Equation (3)). This is based on the following two aspects of observations. (i) The X-ray emission from the jetted TDEs Swift J1644+57 (Bloom et al. 2011; Burrows et al. 2011) and Swift J2058+05 (Cenko et al. 2012) show a plateau lasting for two weeks and then a power-law decay roughly as $L_{\mathrm{X}} \propto t^{-5 / 3}$, roughly tracking the fallback rate. (ii) From the reverberation geometry, one can see that the rise or decay time of the mid-infrared emission from dust provides an upper limit for the duration of the EUV emission. The first and second mid-infrared detections of ASASSN-14li was 35 and 200 rest-frame days (respectively) after discovery or the optical peak (Jiang et al. 2016a), with the flux dropping by a factor of 5 between these two epochs. We can see that the duration of the EUV emission must be $\lesssim 100$ days (the most conservative case is that the mid-infrared flux rose in the first 100 days and then immediately dropped in the second 100 days).

For many other TDEs, the timescale over which the missing energy is released is unconstrained and hence the EUV or jet luminosity is uncertain. For instance, the constraints on the EUV duration from PTF09ge and PTF09axc (van Velzen et al. 2016b) are rather weak, because the mid-infrared observations were made much later. PTF09ge had the first and second detections at 200 and 360 rest-frame days after the optical peak, with roughly equal fluxes between the two epochs. Then, in the most conservative scenario, the duration of the EUV emission may be up to 280 days, which is a weak constraint. We also note that the X-ray emission (associated with the accretion process) from ASASSN-14li had decay time of $~ 30$ days (Miller et al. 2015), while the X-ray emission from ASASSN-15oi steadily rises from
$L_{\mathrm{X}} \sim 5 \times 10^{41} \mathrm{erg} \mathrm{s}^{-1}$ to $5 \times 10^{42} \mathrm{erg} \mathrm{s}^{-1}$ over one year after discovery (Gezari et al. 2017; Holoien et al. 2018). The X-ray light curve from ASASSN-15lh is also qualitatively similar to that from -15oi (Margutti et al. 2017). Since only a small fraction of the total energy released is in the X-ray band $\left(L_{\mathrm{X}} \ll L_{\mathrm{Edd}}\right)$, the diverse X-ray light curves could be due to the variable efficiency of high-energy emission and hence do not provide strong constraints on the circularization or disk-formation timescale.

\section{Summary}

For TDE candidates found by recent surveys, the observed radiation energy in the optical/near-UV bands is only $\sim 10^{51} \mathrm{erg}$, which is much smaller than the total amount of energy generated, $\sim 10^{52}-10^{53} \mathrm{erg}$, if roughly half of the star's mass is accreted onto the $\mathrm{BH}$. In this paper, we show that this energy discrepancy cannot be explained by the radiatively inefficient accretion flow model at super-Eddington accretion rate. We calculated the minimum bolometric energy output from a TDE, based on the assumption that the minimum radiative luminosity from the accretion flow is $\min \left(L_{\mathrm{Edd}}, \eta \dot{M} c^{2}\right)$, where $\eta$ is the radiative efficiency of a standard thin disk and $\dot{M}$ is the mass-feeding rate to the accretion flow. The minimum bolometric energy from our calculation is higher than the observed energy in the optical/ near-UV bands by a factor of 10-100. Therefore, we argue that the missing energy may be in the unobserved EUV band and/or in the form of relativistic jets.

The EUV scenario is supported by the observations that the optical/near-UV spectrum of TDEs is close to the RayleighJeans law and that the luminosity in the X-ray band is at most comparable to that in the optical/near-UV (for thermal TDEs). This scenario is also supported by existing mid-infrared dust reverberation observations, although the quality of currently existing data is relatively low in terms of time and wavelength coverages. Future dust reverberation mapping will not only provide a measurement of the total EUV luminosity from the TDE but also constrain the composition and spatial distribution of dust particles near non-active galactic nuclei.

The jet scenario is disfavored by a number of indirect (and weak) arguments. The strongest constraint is from ASASSN14li, where relativistic off-axis jets with $E_{\mathrm{j}} \sim 10^{52}-10^{53} \mathrm{erg}$ have been ruled out by multiwavelength radio observations. It is currently unclear whether the radio emission from ASASSN14li is representative of all other thermal TDEs, so the jet scenario still remains a viable solution to the energy efficiency puzzle for some TDEs. Future wide field-of-view X-ray transient surveys may directly probe the rate of jetted TDEs and provide better constraints on this scenario.

We also provide evidences that, at least for some TDEs, most of the missing energy (in the EUV and/or in the form of jets) is released within a few times the orbital period of the most tightly bound material $P_{\min }$, which means (1) the circularization of the fallback stream may occur rapidly, and (2) the luminosity of the accretion flow or the jet power may not be capped near the Eddington level when the fallback rate is super-Eddington. For most other TDEs, this energy-release timescale is currently not strongly constrained. In the future, more realistic numerical simulations are needed to understand the detailed circularization and accretion processes.

We thank Sjoert van Velzen and James Guillochon for reading the manuscript and providing valuable comments. The comments from an anonymous referee have improved the 
clarity of the paper. We also thank Tom Holoien, Julian Krolik, Scott Tremaine, Jeremy Goodman, and Brian Metzger for useful discussions. W.L. was supported by the David Alan Benfield Memorial Fellowship at the University of Texas at Austin, and the David and Ellen Lee Fellowship at California Institute of Technology.

\section{ORCID iDs}

Wenbin Lu $\odot$ https://orcid.org/0000-0002-1568-7461

\section{References}

Abramowicz, M. A., \& Fragile, P. C. 2013, LRR, 16, 1

Alexander, K. D., Berger, E., Guillochon, J., Zauderer, B. A., \& Williams, P. K. G. 2016, ApJL, 819, L25

Arcavi, I., Gal-Yam, A., Sullivan, M., et al. 2014, ApJ, 793, 38

Auchettl, K., Guillochon, J., \& Ramirez-Ruiz, E. 2017, ApJ, 838, 149

Begelman, M. C. 1979, MNRAS, 187, 237

Begelman, M. C. 2012, MNRAS, 420, 2912

Blaes, O., Krolik, J. H., Hirose, S., \& Shabaltas, N. 2011, ApJ, 733, 110

Blanchard, P. K., Nicholl, M., Berger, E., et al. 2017, ApJ, 843, 106

Blandford, R. D., \& Begelman, M. C. 1999, MNRAS, 303, L1

Blandford, R. D., \& Begelman, M. C. 2004, MNRAS, 349, 68

Bloom, J. S., Giannios, D., Metzger, B. D., et al. 2011, Sci, 333, 203

Böker, T. 2010, in IAU Symp. 266, Star Clusters: Basic Building Blocks throughout Time and Space, ed. R. de Grijs \& J. R. D. Lépine (Cambridge: Cambridge Univ. Press), 58

Bonnerot, C., Rossi, E. M., Lodato, G., \& Price, D. J. 2016, MNRAS, 455, 2253

Bower, G. C., Metzger, B. D., Cenko, S. B., Silverman, J. M., \& Bloom, J. S. 2013, ApJ, 763, 84

Brown, G. C., Levan, A. J., Stanway, E. R., et al. 2015, MNRAS, 452, 4297

Burrows, D. N., Kennea, J. A., Ghisellini, G., et al. 2011, Natur, 476, 421

Cannizzo, J. K., Lee, H. M., \& Goodman, J. 1990, ApJ, 351, 38

Cenko, S. B., Krimm, H. A., Horesh, A., et al. 2012, ApJ, 753, 77

Cheng, R. M., \& Bogdanović, T. 2014, PhRvD, 90, 064020

Chornock, R., Berger, E., Gezari, S., et al. 2014, ApJ, 780, 44

Coughlin, E. R., \& Begelman, M. C. 2014, ApJ, 781, 82

Coughlin, E. R., \& Nixon, C. 2015, ApJL, 808, L11

Coughlin, E. R., Nixon, C., Begelman, M. C., Armitage, P. J., \& Price, D. J. 2016, MNRAS, 455, 3612

Dai, L., McKinney, J. C., \& Miller, M. C. 2015, ApJL, 812, L39

Dong, S., Shappee, B. J., Prieto, J. L., et al. 2016, Sci, 351, 257

Donley, J. L., Brandt, W. N., Eracleous, M., \& Boller, T. 2002, AJ, 124, 1308

Dou, L., Wang, T., Yan, L., et al. 2017, ApJL, 841, L8

Draine, B. T., \& Lee, H. M. 1984, ApJ, 285, 89

Esquej, P., Saxton, R. D., Komossa, S., et al. 2008, A\&A, 489, 543

Evans, C. R., \& Kochanek, C. S. 1989, ApJL, 346, L13

Generozov, A., Mimica, P., Metzger, B. D., et al. 2017, MNRAS, 464, 2481

Gezari, S., Basa, S., Martin, D. C., et al. 2008, ApJ, 676, 944

Gezari, S., Cenko, S. B., \& Arcavi, I. 2017, ApJL, 851, L47

Gezari, S., Chornock, R., Rest, A., et al. 2012, Natur, 485, 217

Gezari, S., Heckman, T., Cenko, S. B., et al. 2009, ApJ, 698, 1367

Gezari, S., Martin, D. C., Milliard, B., et al. 2006, ApJL, 653, L25

Ghisellini, G., Tavecchio, F., Maraschi, L., Celotti, A., \& Sbarrato, T. 2014, Natur, 515, 376

Giannios, D., \& Metzger, B. D. 2011, MNRAS, 416, 2102

Guhathakurta, P., \& Draine, B. T. 1989, ApJ, 345, 230

Guillochon, J., \& McCourt, M. 2017, ApJL, 834, L19

Guillochon, J., \& Ramirez-Ruiz, E. 2013, ApJ, 767, 25

Guillochon, J., \& Ramirez-Ruiz, E. 2015, ApJ, 809, 166

Hayasaki, K., Stone, N., \& Loeb, A. 2016, MNRAS, 461, 3760

Holoien, T. W.-S., Brown, J. S., Auchettl, K., et al. 2018, MNRAS, 480, 5689

Holoien, T. W.-S., Kochanek, C. S., Prieto, J. L., et al. 2016a, MNRAS, 455,2918

Holoien, T. W.-S., Kochanek, C. S., Prieto, J. L., et al. 2016b, MNRAS, 463,3813

Holoien, T. W.-S., Prieto, J. L., Bersier, D., et al. 2014, MNRAS, 445, 3263

Hung, T., Gezari, S., Blagorodnova, N., et al. 2017a, ApJ, 842, 29
Hung, T., Gezari, S., Cenko, S. B., et al. 2017b, arXiv:1712.04936

Jiang, N., Dou, L., Wang, T., et al. 2016a, ApJL, 828, L14

Jiang, Y.-F., Guillochon, J., \& Loeb, A. 2016b, ApJ, 830, 125

Jiang, Y.-F., Stone, J. M., \& Davis, S. W. 2014, ApJ, 796, 106

Kochanek, C. S. 1994, ApJ, 422, 508

Komossa, S. 2015, JHEAp, 7, 148

Komossa, S., \& Bade, N. 1999, A\&A, 343, 775

Krolik, J., Piran, T., Svirski, G., \& Cheng, R. M. 2016, ApJ, 827, 127

Krolik, J. H., \& Piran, T. 2012, ApJ, 749, 92

Kumar, P., \& Granot, J. 2003, ApJ, 591, 1075

Leloudas, G., Fraser, M., Stone, N. C., et al. 2016, NatAs, 1, 0002

Lodato, G., King, A. R., \& Pringle, J. E. 2009, MNRAS, 392, 332

Loeb, A., \& Ulmer, A. 1997, ApJ, 489, 573

Lu, W., Kumar, P., \& Evans, N. J. 2016, MNRAS, 458, 575

Magorrian, J., \& Tremaine, S. 1999, MNRAS, 309, 447

Mainetti, D., Lupi, A., Campana, S., et al. 2017, A\&A, 600, A124

Margutti, R., Metzger, B. D., Chornock, R., et al. 2017, ApJ, 836, 25

McKinney, J. C., Dai, L., \& Avara, M. J. 2015, MNRAS, 454, L6

McKinney, J. C., Tchekhovskoy, A., Sadowski, A., \& Narayan, R. 2014 MNRAS, 441, 3177

Metzger, B. D., Margalit, B., Kasen, D., \& Quataert, E. 2015, MNRAS, 454, 3311

Metzger, B. D., \& Stone, N. C. 2016, MNRAS, 461, 948

Metzger, B. D., \& Stone, N. C. 2017, ApJ, 844, 75

Miller, J. M., Kaastra, J. S., Miller, M. C., et al. 2015, Natur, 526, 542

Mimica, P., Giannios, D., Metzger, B. D., \& Aloy, M. A. 2015, MNRAS, 450, 2824

Narayan, R., Igumenshchev, I. V., \& Abramowicz, M. A. 2003, PASJ, 55, L69

Narayan, R., \& Yi, I. 1994, ApJL, 428, L13

Ohsuga, K., \& Mineshige, S. 2011, ApJ, 736, 2

Pasham, D. R., Cenko, S. B., Levan, A. J., et al. 2015, ApJ, 805, 68

Peterson, B. M. 1993, PASP, 105, 247

Phinney, E. S. 1989, in IAU Symp. 136, The Center of the Galaxy, ed. M. Morris (Dordrecht: Springer), 543

Piran, T., Sądowski, A., \& Tchekhovskoy, A. 2015a, MNRAS, 453, 157

Piran, T., Svirski, G., Krolik, J., Cheng, R. M., \& Shiokawa, H. 2015b, ApJ, 806,164

Poutanen, J., Lipunova, G., Fabrika, S., Butkevich, A. G., \& Abolmasov, P. 2007, MNRAS, 377, 1187

Rees, M. J. 1988, Natur, 333, 523

Rosswog, S., Ramirez-Ruiz, E., \& Hix, W. R. 2009, ApJ, 695, 404

Roth, N., Kasen, D., Guillochon, J., \& Ramirez-Ruiz, E. 2016, ApJ, 827, 3

Sądowski, A., \& Narayan, R. 2016, MNRAS, 456, 3929

Sądowski, A., Tejeda, E., Gafton, E., Rosswog, S., \& Abarca, D. 2016, MNRAS, 458, 4250

Sari, R., Piran, T., \& Narayan, R. 1998, ApJL, 497, L17

Saxton, R. D., Read, A. M., Esquej, P., et al. 2012, A\&A, 541, A106

Shakura, N. I., \& Sunyaev, R. A. 1973, A\&A, 24, 337

Shen, R.-F., \& Matzner, C. D. 2014, ApJ, 784, 87

Shiokawa, H., Krolik, J. H., Cheng, R. M., Piran, T., \& Noble, S. C. 2015, ApJ, 804,85

Stone, N., Sari, R., \& Loeb, A. 2013, MNRAS, 435, 1809

Stone, N. C., Kesden, M., Cheng, R. M., \& van Velzen, S. 2018, arXiv:1801. 10180

Stone, N. C., \& Metzger, B. D. 2016, MNRAS, 455, 859

Strubbe, L. E., \& Quataert, E. 2009, MNRAS, 400, 2070

Svirski, G., Piran, T., \& Krolik, J. 2017, MNRAS, 467, 1426

Tejeda, E., Gafton, E., Rosswog, S., \& Miller, J. C. 2017, MNRAS, 469, 4483

Ulmer, A. 1999, ApJ, 514, 180

van Velzen, S. 2018, ApJ, 852, 72

van Velzen, S., Anderson, G. E., Stone, N. C., et al. 2016a, Sci, 351, 62

van Velzen, S., \& Farrar, G. R. 2014, ApJ, 792, 53

van Velzen, S., Farrar, G. R., Gezari, S., et al. 2011, ApJ, 741, 73

van Velzen, S., Frail, D. A., Körding, E., \& Falcke, H. 2013, A\&A, 552, A5

van Velzen, S., Mendez, A. J., Krolik, J. H., \& Gorjian, V. 2016b, ApJ, 829, 19

Vinkó, J., Yuan, F., Quimby, R. M., et al. 2015, ApJ, 798, 12

Wang, J., \& Merritt, D. 2004, ApJ, 600, 149

Waxman, E., \& Draine, B. T. 2000, ApJ, 537, 796

Wevers, T., van Velzen, S., Jonker, P. G., et al. 2017, MNRAS, 471, 1694

Wilson, A. S., \& Colbert, E. J. M. 1995, ApJ, 438, 62

Zauderer, B. A., Berger, E., Soderberg, A. M., et al. 2011, Natur, 476, 425

Zubovas, K., Wynn, G. A., \& Gualandris, A. 2013, ApJ, 771, 118 